US Army Corps

of Engineers ${ }_{\circledast}$

Engineer Research and

Development Center

\title{
A Framework and Pilot Tool for the Risk-based Prioritization and Grouping of Nano-enabled Consumer Products
}

Taylor Rycroft, Sabrina Larkin, Alexander Ganin, Treye

August 2021

Thomas, Joanna Matheson, Tessa Van Grack, Xinrong Chen,

Kenton Plourde, Alan Kennedy and Igor Linkov 
The U.S. Army Engineer Research and Development Center (ERDC) solves the nation's toughest engineering and environmental challenges. ERDC develops innovative solutions in civil and military engineering, geospatial sciences, water resources, and environmental sciences for the Army, the Department of Defense, civilian agencies, and our nation's public good. Find out more at www.erdc.usace.army.mil.

To search for other technical reports published by ERDC, visit the ERDC online library at https://erdclibrary.on.worldcat.org/discovery. 


\section{A Framework and Pilot Tool for the Risk-based Prioritization and Grouping of Nano-enabled Consumer Products}

Taylor Rycroft, Sabrina Larkin, Alexander Ganin, Kenton Plourde, and Igor Linkov

Environmental Laboratory

U.S. Army Engineer Research and Development Center

696 Virginia Road

Concord, MA 01746

Alan Kennedy

Environmental Laboratory

U.S. Army Engineer Research and Development Center

3992 Halls Ferry Road

Vicksburg, MS 39180

Treye Thomas, Joanna Matheson, Tessa Van Grack, and Xinrong Chen

U.S. Consumer Product Safety Commission

5 Research Place

Rockville, MD 20850

Final report

Approved for public release; distribution is unlimited.

Prepared for U.S. Army Corps of Engineers

Washington, DC 20314

Under U.S. Consumer Product Safety Commission, Interagency Agreement 


\section{Preface}

This study was conducted for the U.S. Army Corps of Engineers. This work was supported by an interagency agreement from the U.S. Consumer Product Safety Commission.

The work was performed by the Army's Engineer Research and Development Center, Environmental Laboratory (ERDC-EL). At the time of publication of this paper, the Deputy Director of ERDC-EL was Dr. Brandon Lafferty and the Director was Dr. Edmund J. Russo Jr.

This article was originally published online in Environmental Science: Nano on 18 December 2018.

The Commander of ERDC was COL Teresa A. Schlosser and the Director was Dr. David W. Pittman.

DISCLAIMER: The contents of this report are not to be used for advertising, publication, or promotional purposes. Citation of trade names does not constitute an official endorsement or approval of the use of such commercial products. All product names and trademarks cited are the property of their respective owners. The findings of this report are not to be construed as an official Department of the Army position unless so designated by other authorized documents. 


\title{
A framework and pilot tool for the risk-based prioritization and grouping of nano-enabled consumer products
}

\begin{abstract}
The use of engineered nanomaterials (ENMs) in consumer products has expanded rapidly, revealing both in-novative improvements over conventional materials, and the potential for novel risks to human health and the environment. As the number of new nano-enabled products and the volume of toxicity data on ENMs continues to grow, regulatory agencies like the U.S. Consumer Product Safety Commission (CPSC) a small, independent federal agency responsible for protecting consumers from unreasonable risks associated with product use - will require the ability to screen and group a diverse array of nanoenabled consumer prod-ucts based on their potential risks to consumers. Such prioritization would allow efficient allocation of lim-ited resources for subsequent testing and evaluation of high risk products and materials. To enable this grouping and prioritization for further testing, we developed a framework that establishes a prioritization score by evaluating a nano-enabled product's potential hazard and exposure, as well as additional consider-ation of regulatory importance. We integrate the framework into a pilot-version software tool and, using a hypothetical case study, we demonstrate that the tool can effectively rank nano-enabled consumer prod-ucts and can be adjusted for use by agencies with different priorities. The proposed decision-analytical framework and pilot-version tool presented here could enable a regulatory agency like the CPSC to triage reported safety concerns more effectively and allocate limited resources more efficiently.
\end{abstract}

\section{Environmental significance}

There are insufficient resources to comprehensively characterize the health risks of all nano-enabled consumer products and intended use scenarios. Instead, what is needed is a method for prioritizing these products for future risk research and potential mitigation. This article proposes a framework and associated pilot software tool to meet this challenge by effectively ranking nano-enabled products according to their potential human health risk using hazard, exposure, and risk-related user-specified criteria. The findings presented here are generalizable beyond consumers and nano-enabled products, and can be adapted for other chemicals, technologies, and exposure scenarios. This study is environmentally significant because the same nano-enabled consumer products that may pose risks to human health can potentially pose ecological problems if released to the environment.

\section{Introduction}

After a dedicated research effort by the National Nanotechnology Initiative (NNI) in the United States and similar programs worldwide, nanotechnology is being increasingly applied in a variety of industrial sectors. "Nanotechnology," as defined by the NNI, is "the understanding and control of matter at the nanoscale, at dimensions between approximately 1 and 100 nanometers, where unique phenomena enable novel applications". ${ }^{2}$ At this minute scale, a material may exhibit unique physical, chemical, and biological properties different from the individual atoms and molecules that comprise the material, as well as from the bulk form of the matter. ${ }^{3}$ The ability of nanomaterials to achieve specific, desirable properties 
allows researchers and manufacturers to create materials that are stronger, lighter, and more durable, among many other enhancing traits. The integration of nanomaterials in everyday consumer products is particularly prevalent. Nanomaterials employed in cosmetic products can provide improved cleansing and absorption; in food packaging, they can reduce moisture and, as a result, bacteria, which enables the food to stay fresher for longer; in children's toys and blankets, they confer antimicrobial protection; and in polymer composite materials - which comprise consumer products like luggage, power tools, and baseball bats - nanomaterials enable the products to be lighter and more durable. ${ }^{4,5}$

Although the applications of nanomaterials in the consumer product industry are abundant and may offer advantages over conventional materials, possible unintended risks to both human and environmental health and safety need to be considered. The field of nanotechnology research and application is relatively young; while a great deal has been learned since the creation of the NNI in 2001, much is still unknown about the health and safety impact of these materials. ${ }^{6}$ The U.S. government funding for research focused on nanotechnology environmental health and safety concerns (nanoEHS) increased from \$35 million to \$100 million annually over the past 10 years and has cumulatively exceeded \$1 billion from 2006 to 2015 . $^{7}$ Continued investment in nanoEHS demonstrates the still-present need to better identify the EHS risks potentially posed by exposure to nano-enabled products. The NNI has developed a research strategy that outlines key research questions pertaining to human exposures, and uses models to assist in meeting identified data gaps. Recent workshops sponsored by the NNI and the National Nanomanufacturing Network underscored the need to determine and address unknowns in nanomaterial risk. ${ }^{8,9}$ The workshops focused on evaluating gaps in nanomaterial risk management and assessment technologies and highlighting a number of tools being used to share nanomaterial data among researchers and other interested parties. ${ }^{10}$

The integration of nanomaterials into consumer products is occurring at a more rapid rate than the EHS impacts of the nanomaterials in question can be evaluated. As of March 2015, the Nanotechnology Consumer Products Inventory, published by the Project on Emerging Nanotechnologies (an initiative within the Woodrow Wilson International Center for Scholars), contained 1814 consumer products that claimed to contain nanomaterials, a 30 -fold increase from the 54 products listed in 2005. ${ }^{11}$ As a result of such rapid introduction into the marketplace, the need for an efficient method of prioritizing future nanotechnology risk-related research has been identified. In response, the NNI has made a deliberate effort to coordinate nanotechnology-centric EHS research and provide guidance to relevant federal agencies. ${ }^{12,13}$

One U.S. federal agency with a distinct interest in the gap between nanomaterial use and an understanding of a material's health and safety impacts is the U.S. Consumer Product Safety Commission (CPSC). The CPSC is an independent federal regulatory agency that is responsible for protecting the public from unreasonable risks of injury or death associated with the use of more than 15000 types of consumer products within its jurisdiction. ${ }^{14,15}$ Incidents associated with consumer product use, including toys, household chemicals, and power tools, cost the nation $\$ 1$ trillion annually. ${ }^{16}$ While the agency's responsibilities are substantial both in minimizing negative human impact and reducing costs, the CPSCenacted budget in fiscal year 2016 was only \$125 M with a staff of 567 personnel. ${ }^{17}$ These limited resources may be stretched if the volume of reported safety concerns increases as nano-enabled products continue to enter the marketplace.

This paper describes a framework created to assist an agency like the CPSC in grouping products based on their potential risk to consumers and prioritizing subsequent testing and evaluation of the potential human health risk associated with the product use. A transparent and efficient methodology structured with the principles of multicriteria decision analysis allows agencies with limited resources or information to make an educated and justifiable comparative evaluation of multiple consumer products. We describe the integration of the methodology into a Microsoft Excel-based betaversion software tool ("pilot tool") and present a case study illustrating the tool's potential application. We demonstrate that the pilot tool enables a user to screen and prioritize more efficiently a set of nano-enabled consumer products on the basis of risk and the necessity of further testing and evaluation. The framework is not intended to replace formal risk assessment, but rather serves as a useful screening mechanism that flags consumer products and informs the appropriate allocation of limited risk assessment resources.

\section{Methods}

The proposed decision analytic framework, referred to here as the NanoPrioritization Framework (NPF), is based on the estimated human health risk of a product, combined with user-defined criteria designated as important for establishing priorities (referred to as user-defined criteria). The NPF uses multicriteria decision analysis (MCDA), ${ }^{18}$ which allows for the consideration of multiple, complex factors in a decisionmaking process. Rooted in risk and decision science, MCDA tools offer a systematic way of analyzing potentially disparate sources of information and considering risk preferences from multiple stakeholders, such that a list of alternatives can be prioritized. ${ }^{19,20}$

In the following sections, each component of the NPF is described together with its integration into the Excel-based pilot tool. The purpose of developing the pilot tool was to validate the logic of the NPF and provide the foundation for a robust decision-support tool.

\subsection{The NanoPrioritization framework and pilot tool}

The NPF and associated pilot tool were designed to allow a user to assess the three high-level criteria that contribute to a product's prioritization score: hazard potential, exposure potential, and user-defined criteria (Fig. 1). Using MCDA methods, these 


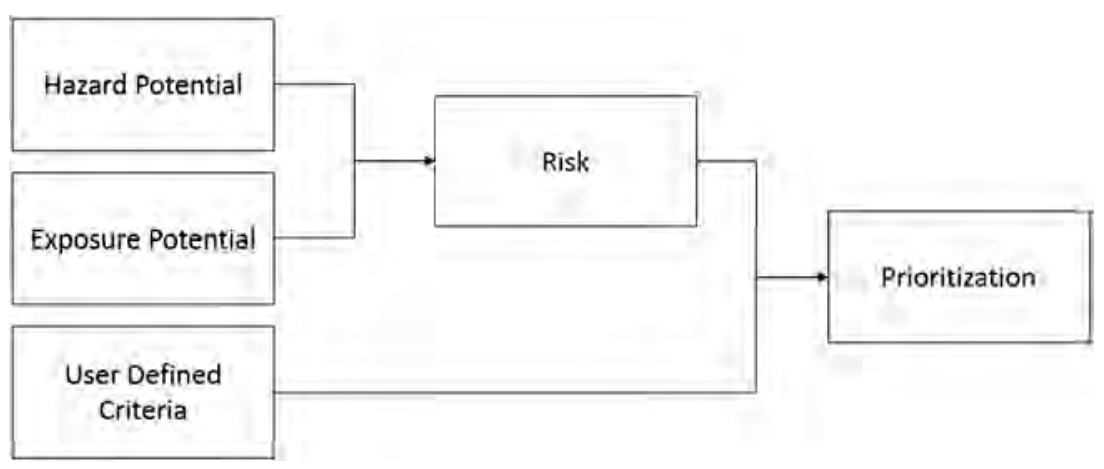

Fig. 1 High-level criteria to establish a prioritization score.

three criteria can be evaluated "bottom-up" using qualitative information provided by the user as sub-criteria, and then translated into a quantitative prioritization score to help assess whether additional research is appropriate.

To derive a quantitative prioritization score, the pilot tool requires the user to provide lower-level, product-specific information that may take the form of qualitative descriptors (e.g., yes/no, high/low) or quantitative values. The user must also indicate the level of uncertainty in their inputs, and in many cases, the weight of importance of the input. The product characteristics, uncertainties, and weights all factor into the rolled-up score for the higher-level criteria (hazard, exposure, and user-defined criteria) and, ultimately, the final prioritization score.

MCDA methods are used to roll-up the lower-level details into higher-level scores. The majority of the scoring within the pilot tool uses the following weighted sum calculation:

$$
P(a)=W_{1} V_{1}\left(a_{i}\right)+\ldots+W_{n} V_{n}\left(a_{n}\right)
$$

where:

$P(a)=$ total prioritization score for product $a$

$W_{i}=$ normalized weight of criterion $C_{i}$ (where $\left.\Sigma W_{i}=1\right)$

$V_{i}\left(a_{i}\right)=$ value of product $a$ reflecting its performance on criterion $C_{i}$

$a_{i}=$ performance score of product $a$ on criterion $C_{i}$ for $i=$ 1 to $n$.

For the few criteria with a multiplicative relationship (i.e. those that are multiplied rather than added to characterize their result), the pilot tool uses the following weighted product calculation:

$$
P(a)=V_{1} a_{1}{ }^{W_{1}} \times \ldots \times V_{n} a_{n}{ }^{W_{n}}
$$

The output prioritization score $P(a)$ ranges from 0 to 100 and is used to compare the priority of different nano-enabled products for further research.

\subsection{High-level criteria}

All user inputs feed into three high-level criteria from which the final prioritization score is calculated: hazard, exposure, and user-defined criteria (Fig. 1). When incorporated into the pilot tool, these three criteria are represented as modules, referred to here as the Hazard module, Exposure module, and User-defined criteria module, respectively. Each of the three modules has defined sub-criteria as described below, and the user is guided to provide their input for each sub-criterion either as qualitative descriptors or quantitative values.

2.2.1 Hazard module. Within the Hazard module, the user must enter information pertaining to the nanomaterial within the consumer product, such as physical dimensions of the nanomaterial, solubility, agglomeration potential, toxicity to specific organs, and other "hazard factors" that may contribute to an adverse health outcome. Characteristics of the bulk form of the nanomaterial must also be recorded, along with the user's degree of uncertainty, or confidence, for each input (measured on a scale of 1-5 where 5 represents highest uncertainty).

The hazard factors in this module are drawn directly from five publicly available control banding tools for nanomaterials. These five tools were designed by government agencies and academia to categorize the hazards of nanomaterials when little quantitative data are available. The tools are:

1. CBNanoTool 2.0 ("CBNanoTool"); ${ }^{21}$

2. Development of specific control banding tool for nanomaterials ("ANSES"); ${ }^{22}$

3. The precautionary matrix for synthetic nanomaterials ("SwissPrecMatrix"); ${ }^{23}$

4. Guidance for working safely with nanomaterials and nanoproducts ("Guidance"); ${ }^{24}$ and

5. A conceptual tool for categorization and communication of exposure potentials and hazards of nanomaterials in consumer products ("NanoRiskCat"). ${ }^{25}$

These tools have been cited in the literature ${ }^{26-30}$ as useful first-tier assessments of emerging nanoscale materials and serve as inputs to the Hazard module through the MCDA hierarchy shown in Fig. 2. The hazard information required by the five tools (e.g. particle shape, particle diameter, surface reactivity, solubility, etc.) is available from sources such as published physicochemical or toxicology literature and nanomaterial safety data sheets (e.g., NanoSustain ${ }^{31}$ ).

The user moves from data provided in the literature (e.g., evidence that the nanomaterial is a reproductive hazard) to 


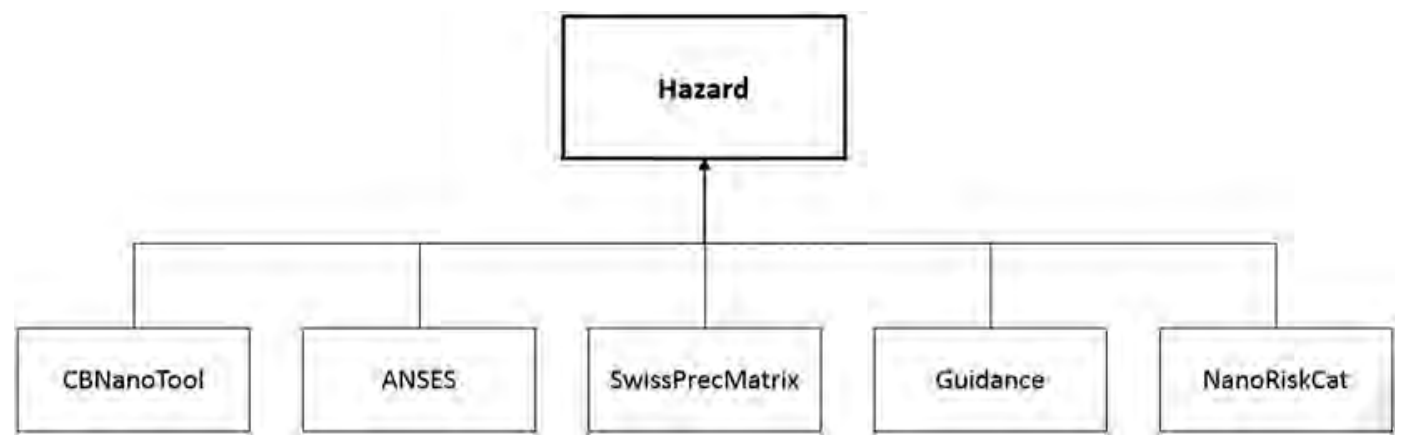

Fig. 2 Hazard module hierarchy.

the input parameter in the Hazard module (e.g., "Is the nanomaterial a reproductive hazard?") using simple drop-downs that offer values consistent with those in the publicly available control banding tools (e.g., yes/maybe/no/unknown). By assembling the hazard factors from each of the five tools in a single module, the Hazard module effectively allows a user to employ the five control banding tools simultaneously. If the user used each tool individually, the outputs generated from the tools may vary by scale (i.e., 1-3 vs. 1-5) or category (i.e., low/medium/high vs. green/yellow/red). Instead, the Hazard module normalizes these outputs and converts them to a 1100 score so that the results from each of the five tools can be integrated into the overall hazard score. For quantitative scale outputs (e.g., 1-5), the score conversion works by dividing the output by the maximum possible value in the scale and then multiplying by 100 , and for the qualitative category outputs the conversion follows the same process but first converts the qualitative statements to quantitative values (e.g., convert low/medium/high to $1 / 2 / 3$ ).

Additionally, the pilot tool allows the user to assign weights to each of the five control banding tools to express the perceived importance of that tool's output. Perceived importance may be affected by the methodology of the control banding tool (e.g., guided scoring vs. decision tree), the tool's targeted at-risk human population (e.g., consumers, factory workers, laboratory researchers), or the level of detail that the tool collects. A user at the CPSC, for example, may choose to assign greater weights to the tools they deem more applicable to consumers, or more robust than the other tools.

2.2.2 Exposure module. In the Exposure module, the user is required to enter information pertaining to the product's exposure factors, such as characteristics of the product's use throughout its life, characteristics of how the product might release nanoscale materials to the consumer, and characteristics of the consumer who will be using the product. These factors contribute to the magnitude of exposure for a single consumer and feed into the overall exposure score through the MCDA hierarchy shown in Fig. 3.

The user must input weights for each sub-criterion (e.g., use environment) based on their evaluation of its importance to the overall exposure score. As in the Hazard module, the user must also express a degree of uncertainty (scale of 1-5) for each input. The user is expected to have some familiarity with the product being assessed and be able to provide a reasonable estimate for the exposure information required (e.g. degree of exposure to UV light, duration of product use, type of product matrix, etc.) which can be partially informed by sources such as the U.S. Environmental Protection Agency's Exposure Factors Handbook. ${ }^{32}$

2.2.3 User-defined criteria module. The third and final module is the User-defined criteria module. The user is prompted to enter information that can influence a product's prioritization score but does not contribute to the risk (hazard and exposure) posed by the product. For a regulatory agency like the CPSC, the sub-criteria within this module may come from the agency's published policies for determining its priorities. For the purposes of this proposed framework and pilot tool, the four top-level sub-criteria in the Userdefined criteria module were partially influenced by the criteria listed in 16 C.F.R. $\S 1009.8(c)$, Policy on establishing priorities for Commission action, which describes eight factors that the CPSC considers when prioritizing action. ${ }^{33}$ The four top-level sub-criteria in the User-defined criteria module are: (1) perception, which refers to consumer views on a product and/or engineered nanomaterial, as well as views of federal agencies and manufacturers; (2) origin, which refers to where the product is from and who made it; (3) product familiarity, which refers to consumer familiarity with the product type and regulator familiarity with the use of the engineered nanomaterial; and (4) vulnerable population, which evaluates whether the product is used by vulnerable populations (e.g., children, elderly, etc.). All user inputs feed into the overall user-defined criteria score through the MCDA hierarchy shown in Fig. 4.

As in the Hazard and Exposure modules, each component of the User-defined criteria module can be weighed based on the user's evaluation of its importance to the overall userdefined criteria score, and uncertainty is recorded. The user is expected to have some familiarity with the product being assessed and be able to provide a reasonable estimate for the information required (e.g. where the product is manufactured, whether consumers are familiar with the potential hazards of the product, etc.) which can be informed by sources such as corporate websites, news releases, or recalls or bans of the product instituted by other nations. 


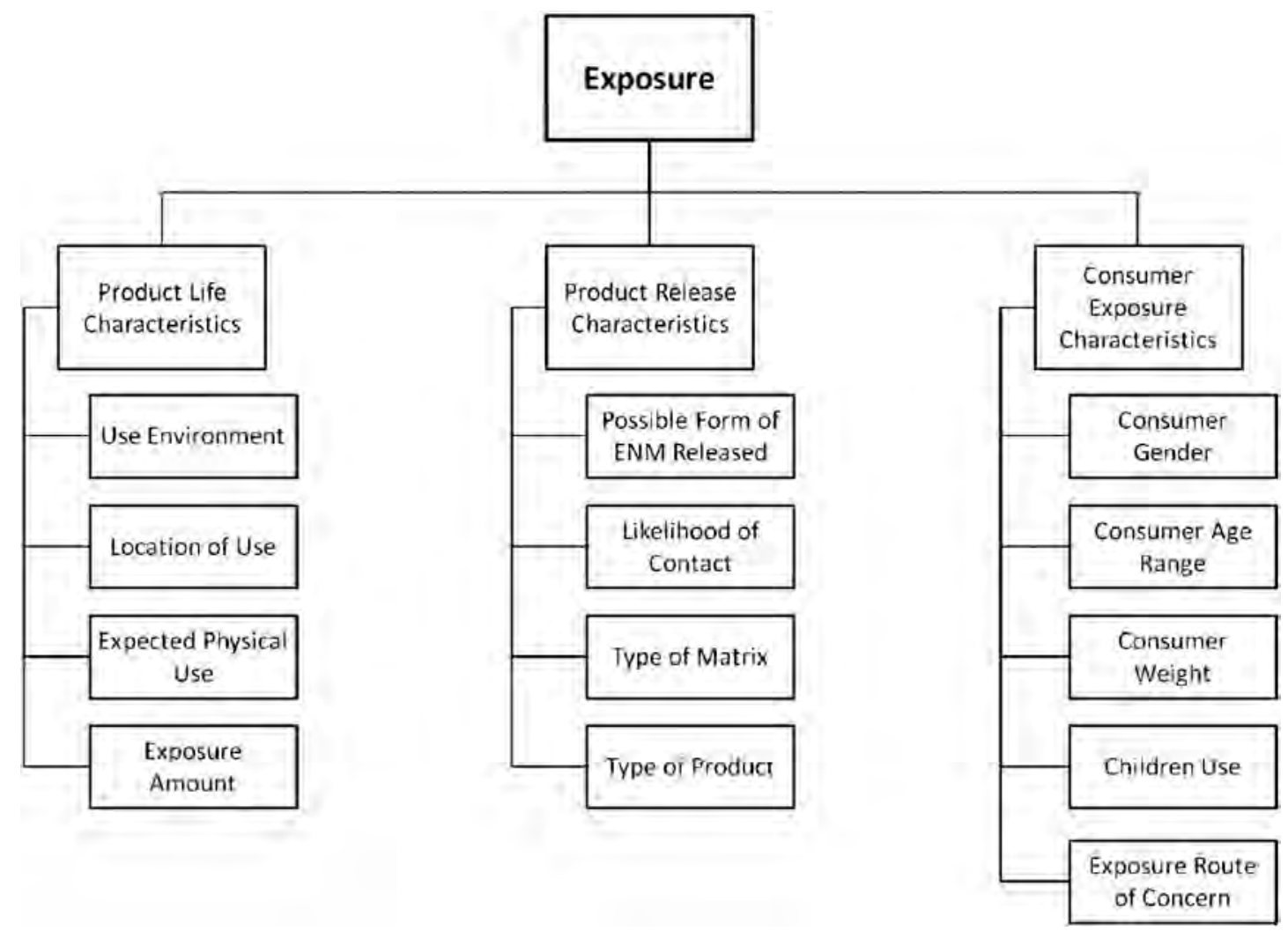

Fig. 3 Exposure module hierarchy.

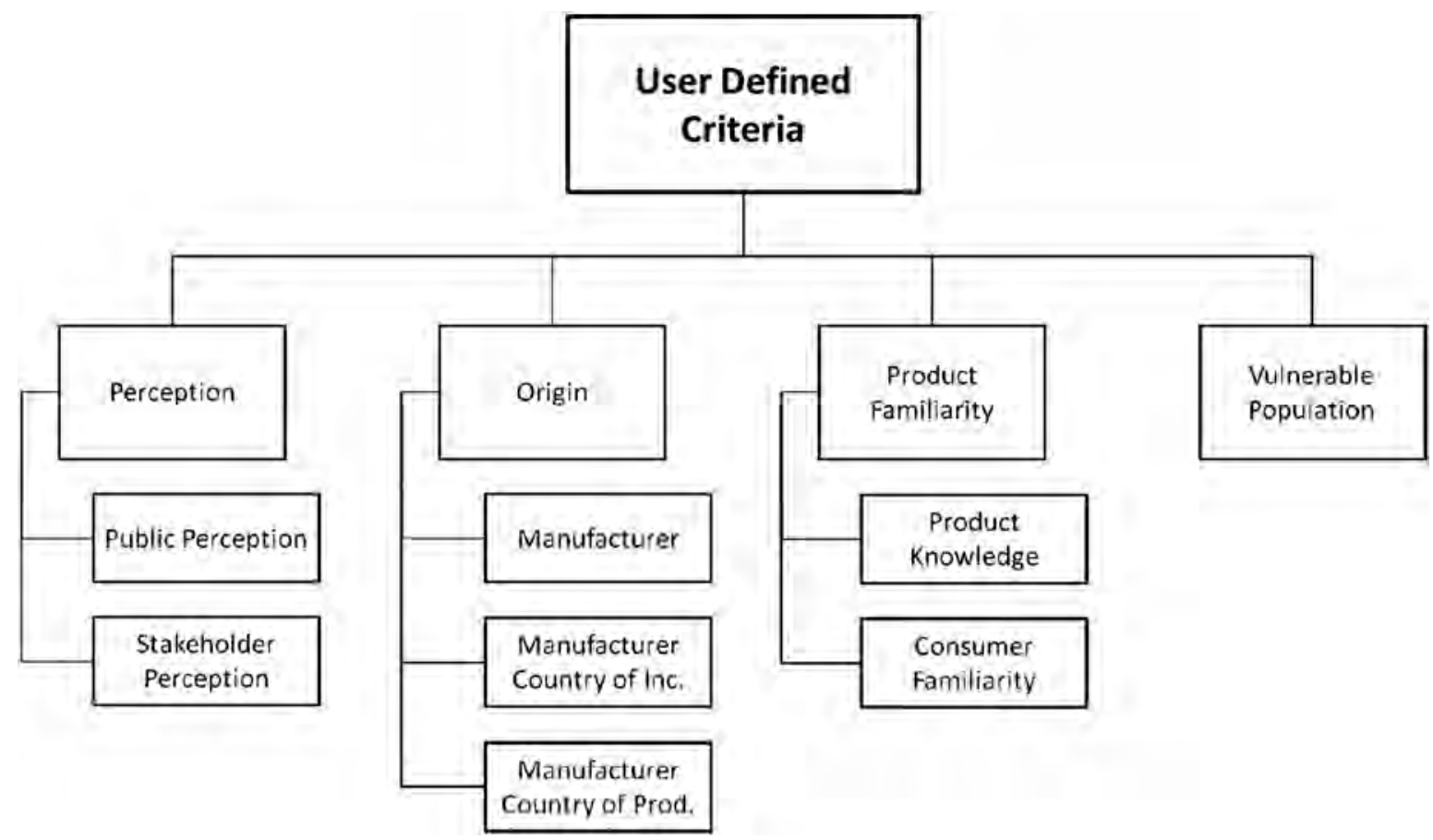

Fig. 4 User-defined criteria module hierarchy.

\subsection{NanoPrioritization framework and pilot tool outputs}

Upon completion of the Hazard, Exposure, and User-defined criteria modules, the user can evaluate the pilot tool results.
Calculations based on the user's inputs in each of the three modules are summarized as raw scores and uncertainty scores. The user assigns weights (perceived importance) to 
the top-level criteria from each module to derive the overall hazard score, exposure score, and user-defined criteria score.

The hazard score and exposure score have a multiplicative relationship and combine (as a weighted product) to generate the risk score (Fig. 1). The user may assign different weights to the hazard score and exposure score, depending on whether they perceive hazard or exposure to be a greater determinant of risk. Once calculated, the Risk Score is combined with the user-defined criteria score, using a weighted sum calculation to generate the overall prioritization score (Fig. 1). Again, the user may choose the weight of the risk score and user-defined criteria score based on whether they view risk or user-defined criteria as a more important contributor to priority for future research.

The overall uncertainty score follows the same mathematical process as the derivation of the overall prioritization score. Uncertainty is specified for each input and then propagates to an uncertainty score for each module using the same weighted sum or weighted product calculations that derive the hazard score, exposure score, and user-defined criteria score. The only variation exists in the Hazard module, where each of the five control banding tools first receive an uncertainty score based on the uncertainty entered for each input and the weight that each input contributes to the score generated by that control banding tool. For example, in CBNanoTool 2.0, solubility can contribute up to $10 \%$ of the hazard score whereas carcinogenicity can only contribute up to $6 \%$, so the uncertainty inputs for those parameters have 0.10 and 0.06 weight applied, respectively, in the weighted sum calculation.

\section{Hypothetical case study}

To demonstrate the pilot tool's ability to prioritize a subset of nano-enabled consumer products, a small case study was performed independently by the U.S. Army Engineer Research and Development Center (ERDC) for seven hypothetical consumer products. Table 1 shows the distinctions made among the seven nano-enabled products (NPs), and Fig. 5 shows the differences in final prioritization score among the four applied weighting schemes. It should be noted that in this hypothetical case, the level of uncertainty for each input was the same across all seven hypothetical NPs, so the final un-

Table 1 Seven hypothetical nano-enabled products

\begin{tabular}{ll}
\hline NP1 & High hazard score, low exposure score \\
NP2 & Low hazard score, high exposure score \\
NP1H & NP1 with high user defined criteria score \\
NP1L & NP1 with low user defined criteria score \\
NP2H & NP2 with high user defined criteria score \\
NP2L & NP2 with low user defined criteria score \\
NP3 & Mostly unknown inputs across modules
\end{tabular}

${ }^{a}$ The pilot tool maintains a health-protective approach throughout the modules by assigning higher scores to the input value "unknown" (e.g., CBNanoTool 2.0 assigns an "unknown" selection a value of $75 \%$ of the maximum score for that input). certainty scores for each NP did not vary and are not depicted here. Although the uncertainty score is not incorporated into the overall prioritization score for an NP, this feature is useful as a tie-breaker between NPs with similar prioritization scores.

Fig. 5 shows the variation in score across the weight schemes. The first weight scheme is the "default," which weighs the risk score (comprised of hazard and exposure) and user-defined criteria score (Fig. 1) equally. The subsequent three weight schemes deviate from the default by placing emphasis on the total risk score, the hazard score, or the exposure score.

When evaluating the initial default scores, NPs with higher user-defined criteria scores (NP1H, NP2H, and NP3) had higher overall scores. The default scenario weights the risk score and the user-defined criteria score equally, meaning that a high hazard score or a high exposure score have less of a potential to influence the total prioritization score than a high user-defined criteria score. This was accounted for in the equal hazard/exposure/user-defined criteria weighting scenario; and while NPs with higher user-defined criteria scores still generally scored higher, the difference between the prioritization scores of these NPs and the other NPs was smaller.

It should also be noted that, although NP1 has a higherthan-average hazard score, and NP2 has a higher-than-average exposure score, the 1:1 weighting in the default setting neutralizes the imbalance. The risk scores of NP1 and NP2 are close together; and having one high component doesn't necessarily result in a high overall prioritization score. This is illustrated more clearly in Fig. 6; while some NPs have higher than average scores in either the hazard or exposure category, their overall risk score remains relatively consistent.

Weighting schemes for the determination of the risk score were altered, as well. The magnitude of the change among the seven NPs when looking at both 2:1 hazard:exposure scenarios and 1:2 hazard:exposure scenarios was similar among each of the seven. However, the direction of change depended on the weighting scenario and which of the two components of the risk score were initially higher (Fig. 7).

Although only a hypothetical illustration, this case study demonstrates that with the NPF incorporated as the backbone of the pilot tool, the prioritization of nano-enabled products is more easily justified, and the nuances contributing to the prioritization are more easily identified and understood. Applied to a larger subset of the 1800+ marketed consumer products that claim to be nano-enabled, a prioritization list, such as the one developed in this case study, can help an agency like the CPSC to more efficiently filter and research the large number of reported adverse events it receives.

\section{Discussion}

The NPF and associated pilot tool allow a user to perform a screening assessment of a consumer product to evaluate its 


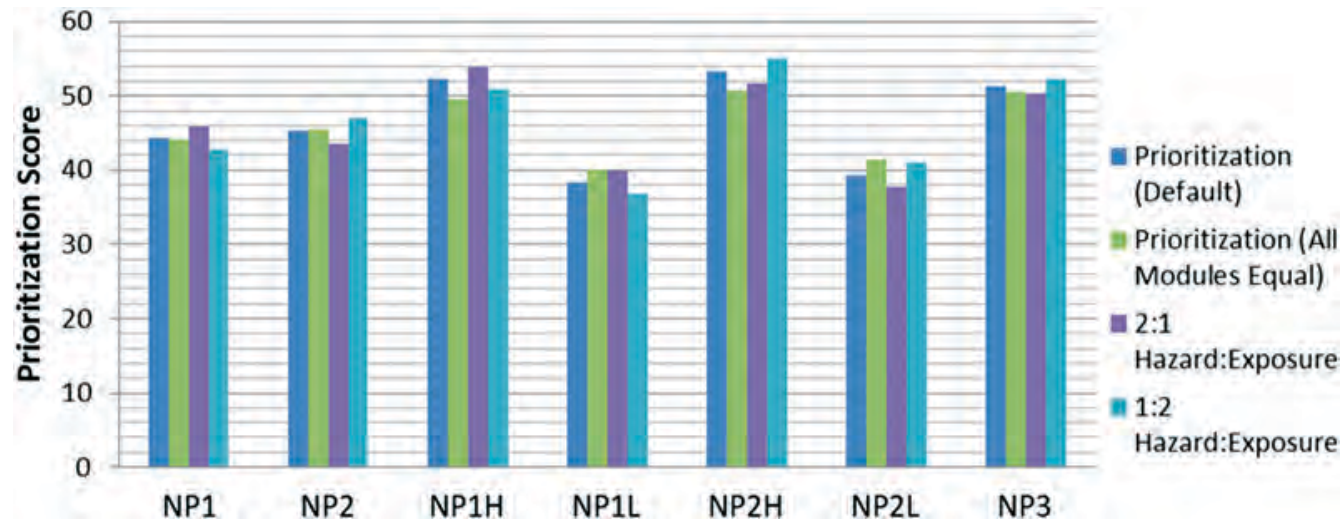

Fig. 5 Prioritization score comparison among different weighting schemes.

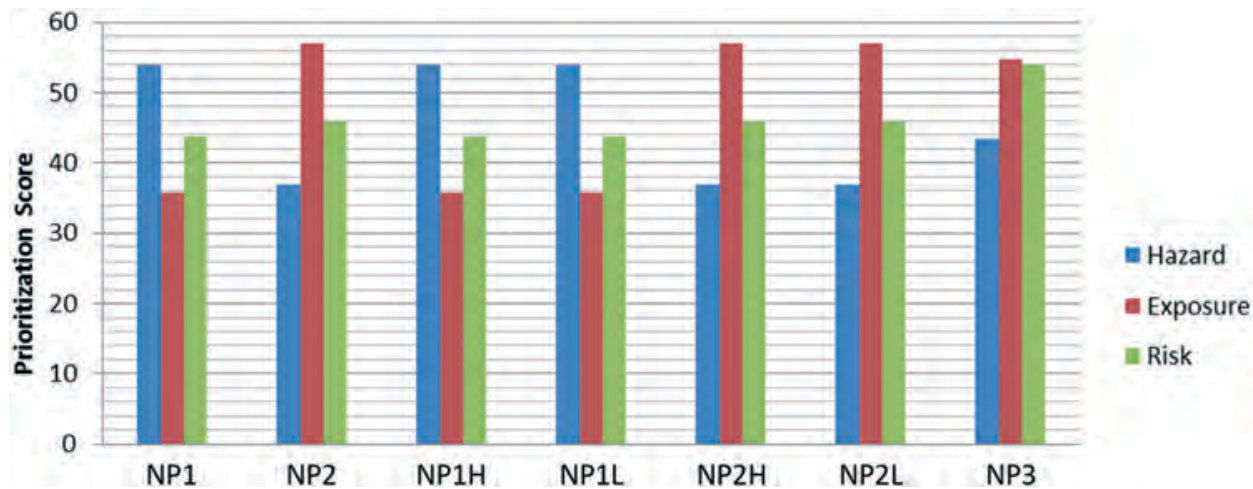

Fig. 6 Hazard, exposure, and combined risk scores (default weighting).

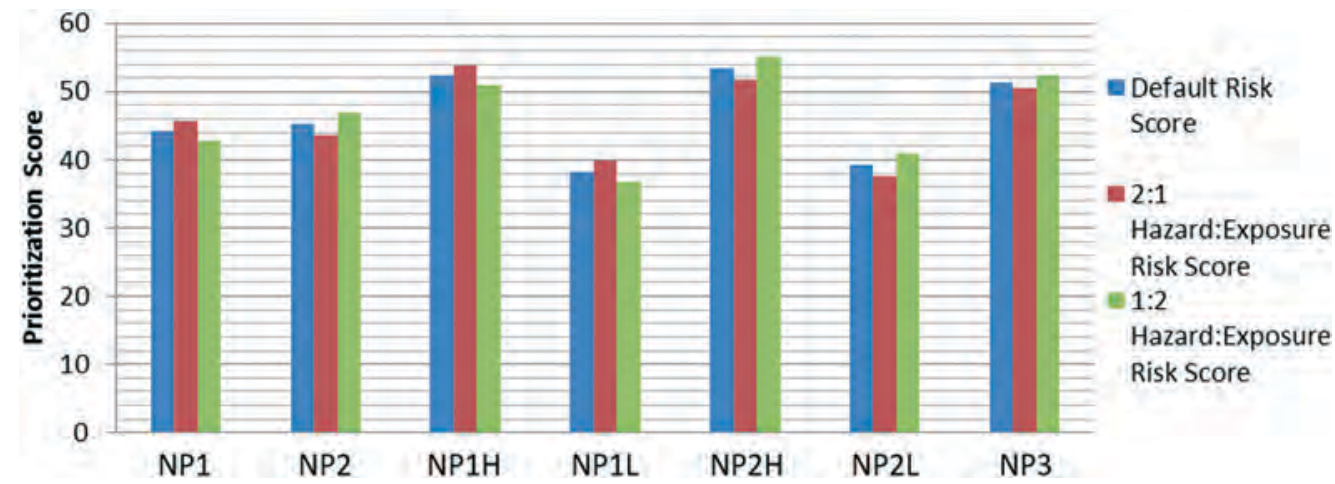

Fig. 7 Comparison of risk scores among different weight schemes.

potential hazard and exposure and its performance against criteria important to the user. The resulting prioritization score for the consumer product can be compared to scores for other consumer products, allowing the user to rank or group products and identify those that warrant additional study. The NPF enables this preliminary investigation of consumer products, despite limited information about the nanomaterial(s) that the product incorporates. The hazard, release capability, and environmental fate and transport of the nanomaterial will likely have some degree of uncertainty, and the pilot tool is able to represent the user's estimate of this uncertainty alongside the overall prioritization score. It must be reiterated and emphasized, though, that evaluation and comparison of consumer products using the NPF is not intended to replace formal risk assessment. Risk assessments require significant time and resources, and the NPF serves as a useful screening mechanism that flags consumer products and informs the appropriate allocation of limited resources for assessing risks and conducting further research to reduce associated uncertainties. 
It should also be noted that in the pilot tool, a user inputs information pertaining to the nanomaterial that may be released from a consumer product. The nanomaterial released and to which a consumer may be exposed, however, often has a different composition from that of the "pristine" nanomaterial used during manufacture of the product. ${ }^{6}$ The fact that nanomaterials may transform as they interact with the product matrix or constituents of the environment is a scientific challenge that is well-documented within the exposure assessment community. ${ }^{34}$ Research shows that the pristine nanomaterial is usually not what is released from a consumer product - typically the consumer is exposed to some combination of the nanomaterial and the product matrix. The toxicity profiles of the pristine and as-released forms of the nanomaterial could be very different. ${ }^{35,36}$ Ideally, the user would know what form the nanomaterial will take as it is released from the product matrix, but the technology, labor, and basic research required to attain this knowledge is currently timeand cost-prohibitive. Thus, users of the NPF will likely continue to make the assumption that the pristine form of the nanomaterial is released, which is likely a conservative assumption when considering nano-specific properties of concern in consumer products. When new information about the actual composition of the released nanomaterials is available, the product can be reassessed for a more accurate depiction of that product's risk to consumers.

The NPF and pilot tool were developed with regulatory agencies like the CPSC in mind, and the pilot tool's potential utility in the consumer product space was demonstrated through the hypothetical case study. To convert the pilot tool into a robust decision-support tool, however, some enhancements should be considered. First, the NPF and pilot tool described here exclusively address the concerns of the CPSC, and therefore, they only consider risk in the use phase of the product life cycle. Risks to the environment, laboratory researchers that develop nanomaterials, and workers who manufacture nano-enabled consumer products are not currently considered. When considering future enhancements to the pilot tool, it would be beneficial to include hazard factors and exposure factors for the occupationally exposed populations and to allow users of the framework to specify which at-risk population they are safeguarding. The proposed framework is generic enough to include all stages of the product life cycle. ${ }^{37}$ Integration of other product life cycle risk could broaden the applicability of the pilot tool to other federal agencies, such as the U.S. Environmental Protection Agency and U.S. Occupational Safety and Health Administration.

Additionally, the pilot tool gives the user flexibility in determining the appropriate weights for the criteria that contribute to the hazard score, exposure score, and user-defined criteria score. While this flexibility is beneficial, it requires the user to have some basis for the weight scheme they select. For example, within the Product Life Characteristics criteria of the Exposure module, the user must weigh four sub-criteria according to their contributions to the Product Life Characteristics score. Similarly, within the Hazard mod- ule, weights must be assigned to the control banding tools that feed into the hazard score on the basis of how relevant and robust a particular tool is for the user's analysis. Even experienced users may struggle to identify how one subcriterion should be valued in comparison to the others without defaulting to an equal weighting scheme. Offering users a second option other than a proportionate default weighting scheme could streamline their process of evaluating and comparing the relative importance of the varied criteria. Expert elicitation, in which a sample of subject matter experts would be surveyed on their preferred weighting iteration, could result in the synthesis of an alternative weighting method for users with a limited background seeking expert perspective. Similarly, the pilot tool gives the user flexibility to select an uncertainty score for each input; offering structured guidance on how to determine the uncertainty would help align interpretations of what uncertainty means and how it should be characterized.

Another consideration identified in the development of the NPF and pilot tool is that, depending on the user, the User-defined criteria module may have fewer sub-criteria for a user to address than the Hazard and Exposure modules (there are no restrictions on the number of sub-criteria in the User-define criteria module). Consequently, the user-defined criteria score will be subject to more dramatic changes when one criterion value is altered. Thus, a change in one input may have an outsized impact on the final prioritization score for a given nanoproduct. In the hypothetical case study presented here, this volatility is particularly influential in the default weighting scheme where the user-defined criteria is given more numeric consideration than the hazard or exposure criteria. To address this sensitivity, a user may consider either including a similar number of sub-criteria in the Userdefined criteria module as the Hazard and Exposure modules, or may adjust the weight scheme for the three modules proportionally to adjust for the disproportionate number of sub-criteria within modules. Despite these limitations, the tool still provides a quantitative level of rationale that is an improvement to the status quo of best professional judgment in an information-poor landscape.

Future work could also include the expansion of the framework to assist users looking to prioritize a more general portfolio of chemical products outside the realm of nanomaterials and nano-enabled products. The inputs would have to be changed to reflect hazard and exposure criteria most vital when considering chemical products and also include a separate module specific to the chemical product user's or tester's additional criteria, akin to the User-defined criteria module in the current model, but the fundamental structure of the model would remain intact.

\section{Conclusion}

With the ever-increasing number of nano-enabled consumer products, a methodology is required to group products based on their potential risks in order to efficiently prioritize their 
testing and evaluation for regulatory decision making. Multicriteria decision analysis has been proposed as a useful platform for nanomaterials ${ }^{38,39}$ and chemicals ${ }^{40}$ prioritization and grouping, but user-friendly tools are required for its practical implementation. The framework developed here provides a foundation for evaluating potential risks associated with product use based on a product's hazard, exposure, and other characteristics deemed a priority to the user, and allows for effective allocation of resources to research and test the highest priority products. Future work could include the use of expert elicitation to obtain alternative weighting scenarios, consideration of how a given nanomaterial interacts with the rest of the consumer product and how that differs from the pristine nanomaterial, and investigation of how to better balance the number of criteria within each module. Eventually, a robust decision-support tool based on this NPF and pilot tool should be developed for a broader suite of chemicals and advanced materials. These approaches will facilitate better and more cost-effective risk governance of emerging technologies. ${ }^{41}$

\section{Acknowledgements}

This work was supported by an interagency agreement from the Consumer Product Safety Commission.

\section{References}

1 Y. Dang, Y. Zhang, L. Fan, H. Chen and M. C. Roco, Trends in worldwide nanotechnology patent applications: 1991 to 2008, J. Nanopart. Res., 2010, 12(3), 687-706.

2 U.S. National Nanotechnology Initiative, What It Is and How It Works http://www.nano.gov/nanotech-101/what (accessed March 14, 2018).

3 J. F. Sargent Jr, Nanotechnology: A policy primer, Congressional Research Service Report Prepared for Members and Committees of Congress, 2012, RL34511.

4 U.S. National Nanotechnology Initiative, Benefits and Applications, http://www.nano.gov/you/nanotechnologybenefits (accessed March 14, 2018).

5 M. E. Quadros, R. Pierson IV, N. S. Tulve, R. Willis, K. Rogers, T. A. Thomas and L. C. Marr, Release of silver from nanotechnology-based consumer products for children, Environ. Sci. Technol., 2013, 47(15), 8894-8901.

6 Z. A. Collier, A. J. Kennedy, A. R. Poda, M. F. Cuddy, R. D. Moser, R. I. MacCuspie, A. Harmon, K. Plourde, C. D. Haines and J. A. Steevens, Tiered guidance for risk-informed environmental health and safety testing of nanotechnologies, J. Nanopart. Res., 2015, 17(3), 1-21.

7 U.S. National Nanotechnology Initiative, Environmental Health and Safety, http://www.nano.gov/you/environmentalhealth-safety (accessed March 14, 2018).
8 T. F. Fadel, J. A. Steevens, T. A. Thomas and I. Linkov, Managing Nanotechnology Risk Management, Nano Today, 2015, 10, 6-10.

9 U.S. National Nanotechnology Initiative, Stakeholder Perspectives on Perception, Assessment, and Management of the Potential Risks in Nanotechnology, http://www.nano.gov/ sites/default/files/pub_resource/2013_nni_r3_workshop_ report.pdf (accessed March 14, 2018).

10 M. E. Bates, S. Larkin, J. M. Keisler and I. Linkov, How decision analysis can further nanoinformatics, Beilstein J. Nanotechnol., 2015, 6(1), 1594-1600.

11 M. E. Vance, T. Kuiken, E. P. Vejerano, S. P. McGinnis, M. F. Hochella, Jr., D. Rejeski and M. S. Hull, Nanotechnology in the real world: Redeveloping the nanomaterial consumer products inventory, Beilstein J. Nanotechnol., 2015, 6, 1769-1780.

12 U.S. National Nanotechnology Initiative, The National Nanotechnology Initiative 2011 Environmental, Health, and Safety Research Strategy, http://www.nano.gov/node/681 (accessed March 14, 2018).

13 U.S. National Nanotechnology Initiative, Progress Review on the Coordinate Implementation of the National Nanotechnology Initiative 2011, http:/www.nano.gov/node/ 1157 (accessed March 14, 2018).

14 U.S. Consumer Product Safety Commission, Recruitment Brochure - Join the Team, http://www.cpsc.gov//PageFiles/ 115212/join.pdf (accessed March 14, 2018).

1516 C.F.R. $\S 1000.1$. The Commission.

16 U.S. Consumer Product Safety Commission, About CPSC, https://www.cpsc.gov/About-CPSC (accessed March 14, 2018).

17 U.S. Consumer Product Safety Commission, Fiscal Year 2017 Performance Budget Request, https:/www.cpsc.gov/s3fspublic/pdfs/blk_pdf_FY2017PerformanceBudgetRequest.pdf (accessed March 14, 2018).

18 I. Linkov and E. Moberg, Multi-criteria decision analysis: environmental applications and case studies, CRC Press, 2011.

19 V. Belton and T. Stewart, Multiple criteria decision analysis: an integrated approach, Springer Science \& Business Media, 2002.

20 R. L. Keeney and H. Raiffa, Decisions with multiple objectives: preferences and value trade-offs, Cambridge university press, 1993.

21 S. Y. Paik, D. M. Zalk and P. Swuste, Application of a pilot control banding tool for risk level assessment and control of nanoparticle exposures, Ann. Occup. Hyg., 2008, 52(6), 419-428.

22 C. Ostiguy, M. Riediker, J. Triolet, P. Troisfontaines and D. Vernez, Development of a specific control banding tool for nanomaterials, French Agency for food, environmental and occupational health and safety (ANSES), 2010.

23 J. Höck, T. Epprecht, E. Furrer, H. Hofmann, K. Höhner, H. Krug, C. Lorenz, L. Limbach, P. Gehr, B. Nowack, M. Riediker, K. Schirmer, B. Schmid, C. Som, W. Stark, C. Studer, A. Ulrich, N. von Götz, A. Weber, S. Wengert and P. Wick, Version 2.1, Guidelines on the Precautionary Matrix for Synthetic Nanomaterials, Federal Office of Public Health and Federal Office for the Environment, Berne, 2011. 
24 R. Cornelissen, F. Jongeneelen, P. Van Broekhuizen and F. Van Broekhuizen, Guidance working safely with nanomaterials and products, the guide for employers and employees, FNV, VNO-NCV and CNV, 2011.

25 S. F. Hansen, K. A. Jensen and A. Baun, NanoRiskCat: a conceptual tool for categorization and communication of exposure potentials and hazards of nanomaterials in consumer products, J. Nanopart. Res., 2014, 16(1), 2195.

26 A. Juric, R. Meldrum and E. N. Liberda, Achieving control of occupational exposures to engineered nanomaterials, J. Occup. Environ. Hyg., 2015, 12(8), 501-508.

27 D. Fleury, G. Fayet, A. Vignes, F. Henry and E. Frejafon, Nanomaterials risk assessment in the process industries: evaluation and application of current control banding methods, in 14. International Symposium on Loss Prevention and Safety Promotion in the Process Industry, AIDIC, Milano, 2013, vol. 31, pp. 949-954.

28 K. Dimou and C. Emond, Nanomaterials, and Occupational Health and Safety-A Literature Review About Control Banding and a Semi-Quantitative Method Proposed for Hazard Assessment, J. Phys.: Conf. Ser., 2017, 838(1), 012020.

29 D. H. Brouwer, Control banding approaches for nanomaterials, Ann. Occup. Hyg., 2012, 56(5), 506-514.

30 D. Hristozov, S. Gottardo, E. Semenzin, A. Oomen, P. Bos, W. Peijnenburg, M. van Tongeren, B. Nowack, N. Hunt, A. Brunelli and J. J. Scott-Fordsmand, Frameworks and tools for risk assessment of manufactured nanomaterials, Environ. Int., 2016, 95, 36-53.

31 NanoSustain, Final Report Summary - NANOSUSTAIN (Development of sustainable solutions for nanotechnology-based products based on hazard characterization and LCA), https:// cordis.europa.eu/result/rcn/146171_en.html (accessed October 30, 2018).

32 United States Environmental Protection Agency, Exposure Factors Handbook: 2011 Edition, Office of Research and Development, Washington, DC, 2011.
3316 C.F.R. $\S 1009.8(c)$. Policy on establishing priorities for Commission action.

34 U.S. National Nanotechnology Coordination Office (NNCO), Quantifying Exposure to Engineered Nanomaterials (QEEN) from Manufactured Products: Addressing Environmental, Health, and Safety Implications, Workshop Proceedings, Arlington, VA, July 7-8, 2015.

35 C. Pang, N. Neubauer, D. Brown, D. Hristozov, T. Fernandes, V. Stone, W. Wolleben and A. Marcomini, Releases from transparent blue automobile coatings containing nanoscale copper phthalocyanine and their effects on J774 A1 macrophages, NanoImpact, 2017, 7, 75-83.

36 J. Gao, M. S. Sepúlveda, C. Klinkhamer, A. Wei, Y. Gao and C. T. Mahapatra, Nanosilver-coated socks and their toxicity to zebrafish (Danio rerio) embryos, Chemosphere, 2015, 119, 948-952.

37 I. Linkov, B. T. Trump, B. A. Wender, T. P. Seager, A. J. Kennedy and J. M. Keisler, Integrate life-cycle assessment and risk analysis results, not methods, Nat. Nanotechnol., 2017, 12, 740 .

38 I. Linkov, K. Satterstrom, J. Steevens, E. Ferguson and R. Pleus, Multi-criteria decision analysis and environmental risk assessment for nanomaterials, J. Nanopart. Res., 2007, 9, 543-554.

39 V. Subramanian, E. Semenzin, D. Hristozov, E. Zondervanvan den Beuken, I. Linkov and A. Marcomini, Decision Analytic Tools for Sustainable Nanotechnology, Environment Systems and Decisions, 2015, 35, 29-41.

40 J. Mitchell, N. Pabon, Z. A. Collier, P. P. Egeghy, E. CohenHubal, I. Linkov and D. A. Vallero, A Decision Analytic Approach to Exposure-Based Chemical Prioritization, PLoS One, 2013, 8, e70911.

41 T. Rycroft, B. Trump, K. Poinsatte-Jones and I. Linkov, Nanotoxicology and nanomedicine: making development decisions in an evolving governance environment, J. Nanopart. Res., 2018, 20(2), 52. 


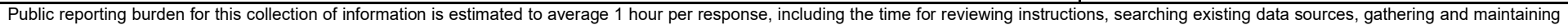

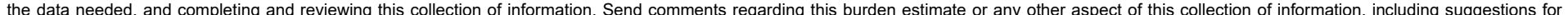

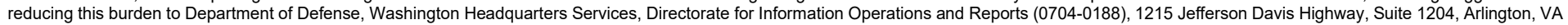

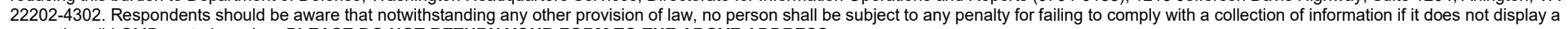
currently valid OMB control number. PLEASE DO NOT RETURN YOUR FORM TO THE ABOVE ADDRESS.
1. REPORT DATE (DD-MM-YYYY)
August 2021
2. REPORT TYPE Final

4. TITLE AND SUBTITLE

A Framework and Pilot Tool for the Risk-based Prioritization and Grouping of

Nano-enabled Consumer Products

\section{AUTHOR(S)}

Taylor Rycroft, Sabrina Larkin, Alexander Ganin, Treye Thomas, Joanna Matheson, Tessa Van Grack, Xinrong Chen, Kenton Plourde,

Alan Kennedy and Igor Linkov

\section{PERFORMING ORGANIZATION NAME(S) AND ADDRESS(ES)}

See next page.
3. DATES COVERED (From - To)

\section{5a. CONTRACT NUMBER}

5b. GRANT NUMBER

5c. PROGRAM ELEMENT NUMBER

\section{5d. PROJECT NUMBER}

5e. TASK NUMBER

\section{5f. WORK UNIT NUMBER}

8. PERFORMING ORGANIZATION REPORT NUMBER

ERDC/EL MP-21-8

10. SPONSOR/MONITOR'S ACRONYM(S)

USACE

11. SPONSOR/MONITOR'S REPORT NUMBER(S)

\section{DISTRIBUTION / AVAILABILITY STATEMENT}

Approved for public release; distribution is unlimited.

13. SUPPLEMENTARY NOTES

This article was originally published online in Environmental Science: Nano 18 December 2018.

This work was supported by an interagency agreement with the U.S. Consumer Product Safety Commission.

\section{ABSTRACT}

The use of engineered nanomaterials (ENMs) in consumer products has expanded rapidly, revealing both innovative improvements over conventional materials, and the potential for novel risks to human health and the environment. As the number of new nanoenabled products and the volume of toxicity data on ENMs continues to grow, regulatory agencies like the U.S. Consumer Product Safety Commission (CPSC) - a small, independent federal agency responsible for protecting consumers from unreasonable risks associated with product use - will require the ability to screen and group a diverse array of nano-enabled consumer products based on their potential risks to consumers. Such prioritization would allow efficient allocation of limited resources for subsequent testing and evaluation of high-risk products and materials. To enable this grouping and prioritization for further testing, we developed a framework that establishes a prioritization score by evaluating a nano-enabled product's potential hazard and exposure, as well as additional consideration of regulatory importance. We integrate the framework into a pilot version software tool and, using a hypothetical case study, we demonstrate that the tool can effectively rank nano-enabled consumer products and can be adjusted for use by agencies with different priorities. The proposed decision-analytical framework and pilot-version tool presented here could enable a regulatory agency like the CPSC to triage reported safety concerns more effectively and allocate limited resources more efficiently.

\section{SUBJECT TERMS}

Nanostructured materials - Analysis, Nanostructured materials - Toxicology, Nanostructured materials - Health aspects, Nanostructured materials - Environment aspects, Nanostructured materials - Research, Product safety

\section{SECURITY CLASSIFICATION OF:}

\section{a. REPORT}

Unclassified

b. ABSTRACT
Unclassified

17. LIMITATION OF ABSTRACT

\section{c. THIS PAGE}

Unclassified
18. NUMBER OF PAGES

UU 19a. NAME OF RESPONSIBLE PERSON

19b. TELEPHONE NUMBER (include area code) 
7. PERFORMING ORGANIZATION NAME(S) AND ADDRESS(ES)

Environmental Laboratory

U.S. Army Engineer Research and Development Center 3992 Halls Ferry Road

Vicksburg, MS 39180 and Concord, MA 01746

U.S. Consumer Product Safety Commission

5 Research Place

Rockville, MD 20850 